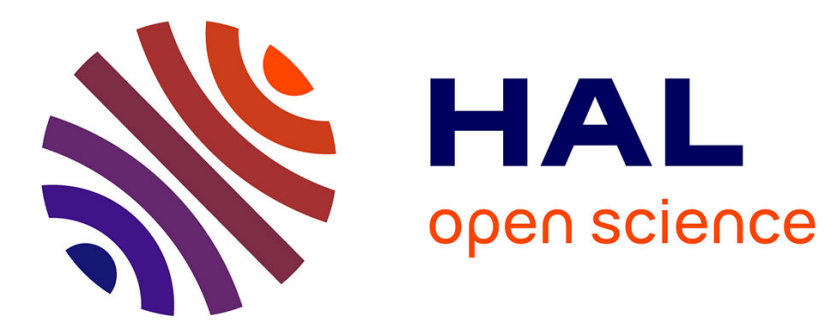

\title{
On the Probability Distributions of Spectrogram Coefficients for Correlated Gaussian Process
}

\author{
Julien Huillery, Fabien Millioz, Nadine Martin
}

\section{To cite this version:}

Julien Huillery, Fabien Millioz, Nadine Martin. On the Probability Distributions of Spectrogram Coefficients for Correlated Gaussian Process. 2006, pp. III-436-439. hal-00085152

\section{HAL Id: hal-00085152 \\ https://hal.science/hal-00085152}

Submitted on 11 Jul 2006

HAL is a multi-disciplinary open access archive for the deposit and dissemination of scientific research documents, whether they are published or not. The documents may come from teaching and research institutions in France or abroad, or from public or private research centers.
L'archive ouverte pluridisciplinaire HAL, est destinée au dépôt et à la diffusion de documents scientifiques de niveau recherche, publiés ou non, émanant des établissements d'enseignement et de recherche français ou étrangers, des laboratoires publics ou privés. 


\title{
ON THE PROBABILITY DISTRIBUTIONS OF SPECTROGRAM COEFFICIENTS FOR CORRELATED GAUSSIAN PROCESS
}

\author{
Julien HUILLERY, Fabien MILLIOZ and Nadine MARTIN \\ Image and Signal processing Laboratory (LIS) \\ 961 rue de la Houille Blanche, BP 46 \\ 38402 St Martin d'Heres Cedex. France \\ Julien.huillery@lis.inpg.fr, nadine.martin@lis.inpg.fr
}

\begin{abstract}
This paper deals with the probability distribution of spectrogram coefficients related to a correlated centered Gaussian process. It is shown that the windowing operation and the presence of correlation between input samples may introduce heteroscedaticity and correlation between the real and imaginary parts of the Short Time Fourier Transform. The impact of this phenomenon on spectrogram distribution is evaluated in terms of deviation from the chi-square distribution. A numerical method to calculate the probability density function of the spectrogram coefficients is provided and deviation from the chi-square distribution is evaluated using the KullbackLiebler divergence. This measure of deviation is used to control the validity of a chi-square approximation.
\end{abstract}

\section{INTRODUCTION}

The spectrogram is a widely used time-frequency representation for the analysis of multi-components non-stationary signals. As a main advantage, it does not display any interference terms as opposed with other quadratic time-frequency representations like the Wigner-Ville distribution. However, this feature is counterbalanced by a loss of energy concentration. When the signal under investigation is random, its spectrogram is a random field, and the energy at every timefrequency location is distributed as a particular probability law. In a time-frequency detection context, we have to decide if a given time-frequency location contains energy that originates from the signal or not. The determination of the spectrogram probability laws corresponds to the first step for the use of probabilistic approaches such as Bayesian ones.

In this paper we focus on the probability distribution of spectrogram coefficients related to a centered stationary Gaussian process with covariance matrix $\mathbf{R}$. No hypothesis is made on the whiteness of the process and $\mathbf{R}$ is thus not necessarily diagonal. From a detection point of view, the case under study corresponds to the null hypothesis $H_{0}$ where only Gaussian noise is present at the investigated time-frequency location. Probability distributions of spectral estimates using pe- riodogram have already been studied for correlated centered Gaussian process [1][2][3]. The study presented here extends the results in two ways. Firstly, previous works make no references about the impact of the nature of the analysis window that we found non negligible. Secondly, the probability distributions are given in terms of the generalized hypergeometric function, that may be expanded into a sum of exponential functions [3]. These expressions are not easily practicable for theoretical developments. We evaluate here the possibility to approximate the spectrogram coefficient distributions with the well-defined $\chi^{2}$ law [4] that is known to be the exact distribution for an uncorrelated Gaussian process.

We investigate the probabilities of spectrogram coefficients defined as the squared modulus of the signal Short-Time Fourier Transform (STFT). In section 2 we show how second order statistical characteristics of the input process and windowing operation intervene in the STFT probability distribution. Section 3 proposes a simple way to compute the probability density function of the squared modulus of a Gaussian vector. Deviation from the $\chi^{2}$ distribution is then evaluated using the Kullback-Liebler Divergence. In section 4, we propose an illustration of the impact of the analysis window's nature for an exponentially correlated Gaussian sequence and finally discuss the validity of a $\chi^{2}$ approximation in section 5 .

\section{PROBABILITY DISTRIBUTION OF STFT REAL AND IMAGINARY PARTS}

Given a discrete analysis window $w[n]$ of length $M$, the ShortTime Fourier Transform (STFT) $X_{w}[n, k]$ of a discrete signal $x[n]$ is formed by the successive Discrete Fourier Transforms of the windowed signal. The spectrogram $S_{x}^{w}[n, k]$ corresponds to the squared modulus of the STFT or equivalently to the sum of the squares of the STFT real and imaginary parts, $X_{w}^{r}[n, k]$ and $X_{w}^{i}[n, k]$ respectively. We thus start from the following definitions:

$$
S_{x}^{w}[n, k]=X_{w}^{r}[n, k]^{2}+X_{w}^{i}[n, k]^{2},
$$




$$
\begin{aligned}
X_{w}^{r}[n, k] & =\sum_{m=0}^{M-1} x[n-m] w[m] \cos \left(-2 \pi k \frac{m}{M}\right), \\
X_{w}^{i}[n, k] & =\sum_{m=0}^{M-1} x[n-m] w[m] \sin \left(-2 \pi k \frac{m}{M}\right) .
\end{aligned}
$$

In the sequel, we consider the random vector

$$
\mathbf{X}[n, k]^{T}=\left[X_{w}^{r}[n, k] X_{w}^{i}[n, k]\right] .
$$

Equations (2) and (3) express the real and imaginary parts of the STFT as linear combinations of signal samples. As the $x[n]$ are centered Gaussian variables (not necessarily independent), $X_{w}^{r}[n, k]$ and $X_{w}^{i}[n, k]$ are also distributed as centered Gaussian law. $\mathbf{X}[n, k]$ is thus a zero-mean Gaussian vector defined by three parameters $\sigma_{r}^{2}, \sigma_{i}^{2}$ and $\sigma_{r i}^{2}$ (for clarity of notation we drop the time-frequency location $[n, k]$ in the probability parameter notations):

$$
\mathbf{X}[n, k]=\left(\begin{array}{c}
X_{w}^{r}[n, k] \\
X_{w}^{i}[n, k]
\end{array}\right) \sim \mathcal{N}\left\{\left(\begin{array}{c}
0 \\
0
\end{array}\right),\left(\begin{array}{cc}
\sigma_{r}^{2} & \sigma_{r i}^{2} \\
\sigma_{r i}^{2} & \sigma_{i}^{2}
\end{array}\right)\right\} .
$$

From equations (2) and (3), we obtain

$$
\begin{gathered}
\sigma_{r}^{2}=W^{T} C_{k} \mathbf{R} C_{k} W \\
\sigma_{i}^{2}=W^{T} S_{k} \mathbf{R} S_{k} W \\
\sigma_{r i}^{2}=W^{T} C_{k} \mathbf{R} S_{k} W
\end{gathered}
$$

where $W$ is the analysis window vector and $C_{k}$ (resp. $S_{k}$ ) is the cosine (resp. sine) diagonal matrix,

$$
\begin{gathered}
W^{T}=[w[0] \cdots w[M-1]] \\
C_{k}=\operatorname{diag}\left[\cos \left(-2 \pi k \frac{m}{M}\right)\right]_{m=0, M-1} \\
S_{k}=\operatorname{diag}\left[\sin \left(-2 \pi k \frac{m}{M}\right)\right]_{m=0, M-1}
\end{gathered}
$$

\section{PROBABILITY DISTRIBUTION OF A GAUSSIAN SQUARED MODULUS}

From equation (1), the distribution of spectrogram coefficients $S_{x}^{w}[n, k]$ corresponds to the distribution of the squared modulus of the 2D Gaussian vector $\mathbf{X}[n, k]$. This section first presents a simple way, based on geometrical considerations, for the calculus of such a probability distribution in a general case. Deviation from the chi-squared distribution is then evaluated using the Kullback-Liebler divergence.

\subsection{Numerical calculus of the probability density func- tion of a Gaussian squared modulus}

Let

$$
G=\left(\begin{array}{c}
G_{1} \\
G_{2}
\end{array}\right) \sim \mathcal{N}\left\{\left(\begin{array}{c}
m_{1} \\
m_{2}
\end{array}\right),\left(\begin{array}{cc}
\sigma_{1}^{2} & \rho \sigma_{1} \sigma_{2} \\
\rho \sigma_{1} \sigma_{2} & \sigma_{2}^{2}
\end{array}\right)\right\}
$$
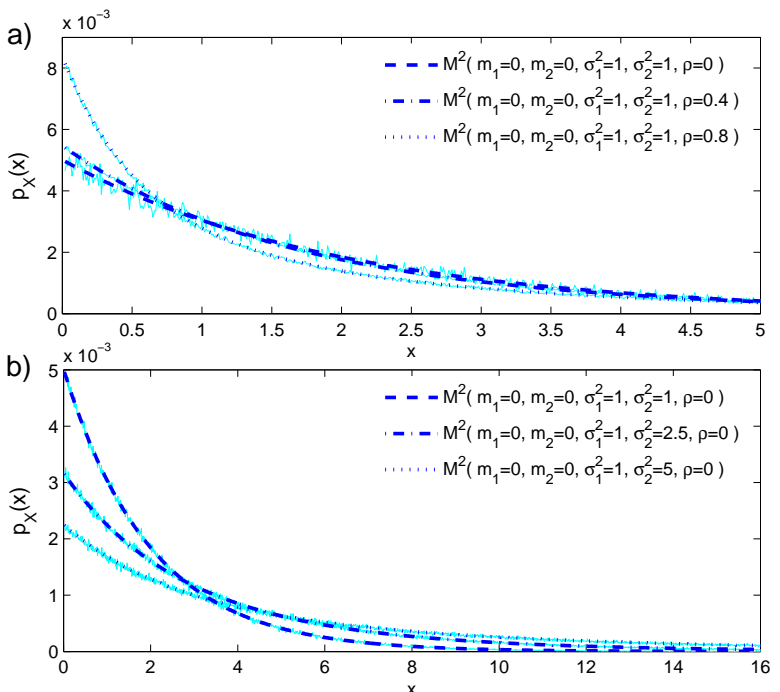

Fig. 1. Central Gaussian squared modulus distributions and corresponding experimental histograms for a) different correlation coefficients and b) different ratio of variances. In each case the dashed line corresponds to the central $\chi^{2}$ distribution

be a $2 \mathrm{D}$ Gaussian vector and $\mathcal{M}^{2}\left(m_{1}, m_{2}, \sigma_{1}^{2}, \sigma_{2}^{2}, \rho\right)$ the distribution of its squared modulus $X=G_{1}^{2}+G_{2}^{2}$, expressed in terms of its five parameters.

When $\sigma_{1}^{2}=\sigma_{2}^{2}=\sigma^{2}$ and $\rho=0, X$ is proportional to a chi-squared variable with two degrees of freedom and noncentrality parameter $m_{1}^{2}+m_{2}^{2}$. For other conditions, $X$ deviates from a $\chi^{2}$ variable and may be identify as a quadratic form in Gaussian variables $X=G^{T} I_{2} G$ where $I_{2}$ is the $2 \times 2$ identity matrix. Distributions of this kind have been extensively studied in the past [5]. Expression of the characteristic function of $X$ are given in terms of the singular values of the covariance matrix of $G$. However the Fourier inversion leading to the probability density function remains difficult.

Here we propose a geometrical approach to compute the probability density function (pdf) of the Gaussian squared modulus $X$. This approach avoids the use of singular value decomposition and Fourier inversion and has revealed computationally very efficient. Looking in the $\left(G_{1}, G_{2}\right)$ plan, the pdf $p_{G}\left(g_{1}, g_{2}\right)$ of vector $G$ is a bidimensional Gaussian distribution. The probability $P[X=x]$ that $X$ equals a given value $x$ corresponds to the probability $P\left[G_{1}^{2}+G_{2}^{2}=x\right]$. The density of probability $p_{X}(x)$ thus corresponds to the density of probability of $G$ over the circle centered at $(0,0)$ with radius $\sqrt{x}$. To adapt the notation to the problem's geometry, we express the pdf of $G$ in cylindrical coordinates $\left(p_{G}\left(g_{1}, g_{2}\right) \rightarrow p_{G}(r, \theta)\right.$ ). This leads to the use of an unique integration on the angular coordinate over $[02 \pi]$ for the calculation of the pdf $p_{X}(x)$ of $X$. That is:

$$
p_{X}(x)=\int_{0}^{2 \pi} p_{G}(\sqrt{x}, \theta) d \theta
$$




$$
\begin{gathered}
\text { where } p_{G}(r, \theta)=\frac{1}{2 \pi \sigma_{1} \sigma_{2} \sqrt{1-\rho^{2}}} \exp ^{-\frac{1}{2\left(1-\rho^{2}\right)} A(r, \theta)}, \\
A(r, \theta)=\left[\frac{\left(r \cos \theta-m_{1}\right)^{2}}{\sigma_{1}^{2}}-\frac{2 \rho\left(r \cos \theta-m_{1}\right)\left(r \sin \theta-m_{2}\right)}{\sigma_{1} \sigma_{2}}+\frac{\left(r \sin \theta-m_{2}\right)^{2}}{\sigma_{2}^{2}}\right] .
\end{gathered}
$$

Spectrogram coefficient distributions for a correlated centered Gaussian process correspond to the central case $m_{1}=$ $m_{2}=0$ with $\sigma_{1}^{2}=\sigma_{r}^{2}$ and $\sigma_{2}^{2}=\sigma_{i}^{2}$. Figure 1 presents some centered Gaussian Squared Modulus (GSM) distributions for different values of the correlation coefficient (figure $1-a)$ ) and ratio of variances $\sigma_{1}^{2} / \sigma_{2}^{2}$ (figure 1-b)). For each distribution, the histogram of $10^{6}$ realisations of the corresponding variable is also plotted so as to validate the accuracy of the method. We may note that heteroscedaticity (difference of variances) tends to stretch the distribution along the quantil axis, whereas correlation acts in the opposite way and tighten the distribution near zero.

\subsection{Deviation from a chi-square distribution}

As mentioned above, when the components of vector $G$ have the same variance and are decorrelated, the Gaussian squared modulus $X$ is proportional to a $\chi^{2}$ variable. We are here interested in the evaluation of the departure from this probability distribution as heteroscedaticity and correlation increase between the vector's components. For this purpose, we calculate the Kullback-Liebler Divergence of the central GSM distribution from the central $\chi^{2}$ distribution as a function of the ratio of variances $\sigma_{1}^{2} / \sigma_{2}^{2}$ and correlation coefficient $\rho$. For a given value of these parameters, we compute a set of $10^{6}$ realisations of the corresponding centered Gaussian vector $G$. From this set we estimate by a near maximum likelihood method [6] the degree of freedom $\delta$ and proportionality coefficient $\alpha$ of the nearest central $\chi^{2}$ distribution. The Kullback-Liebler Divergence $\mathcal{J}\left(\mathcal{M}^{2} \| \chi^{2}\right)$ of the GSM distribution from the $\chi^{2}$ distribution is then calculated as

$$
\mathcal{J}\left(\mathcal{M}^{2} \| \chi^{2}\right)=\int_{0}^{+\infty} p_{\mathcal{M}^{2}}(x) \log \left[\frac{p_{\mathcal{M}^{2}}(x)}{p_{\chi^{2}}(x)}\right] d x .
$$

Results are reported in figure 2. Figures 2-b) and 2-c) show the KL-divergence profiles at $\rho=0$ and $\sigma_{1}^{2}=\sigma_{2}^{2}$ respectively. We can observe that the GSM distribution is highly similar to a $\chi^{2}$ distribution when the ratio of variances does not exceed 1.5 or when the linear correlation coefficient lies between -0.2 and 0.2 .

\section{IMPACT OF WINDOWING FOR AN EXPONENTIALLY CORRELATED PROCESS}

For a centered process, the probability distribution of the spectrogram is defined by the second order statistical characteristics of the STFT vector $\mathbf{X}[n, k]$, namely $\sigma_{r}^{2}, \sigma_{i}^{2}$ and $\sigma_{r i}^{2}$. In section 2, we showed how these characteristics depend on the analysis window $w[n]$ and the covariance matrix $\mathbf{R}$ of the signal (see equations (4), (5) and (6)). In this section, the impact

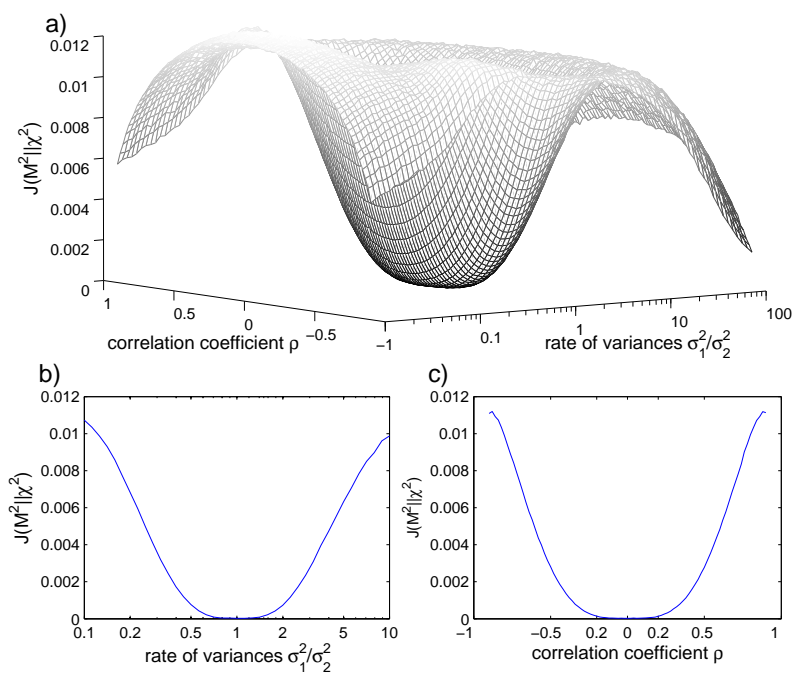

Fig. 2. a) Kullback-Liebler Divergence of the GSM law from the $\chi^{2}$ distribution as a function of ratio of variances $\sigma_{1}^{2} / \sigma_{2}^{2}$ and correlation coefficient $\rho$. b) KL-Divergence profile at $\rho=$ 0 . c) KL-Divergence profile at $\sigma_{1}^{2}=\sigma_{2}^{2}$.

of the nature and length of the window used for the Fourier analysis of correlated process is evaluated. We use an exponentially correlated process, or Ornstein-Uhlenbeck noise, whose autocorrelation function is

$$
\Gamma(t)=\Gamma_{0} \exp \left(-\frac{|t|}{\tau_{c}}\right)
$$

where $\Gamma_{0}$ is set to 1 in this experiment and $\tau_{c}$ is the correlation time in the process. We compute the ratio of the STFT real and imaginary part variances $\sigma_{r}^{2} / \sigma_{i}^{2}$ and their linear correlation coefficient $\rho=\sigma_{r i}^{2} / \sigma_{r} \sigma_{i}$ along frequencies using different types of analysis window. Results are only reported for the rectangular and Hanning windows. The other windows we used (Kaiser, Blackman, Gaussian and Hamming) give similar results as the Hanning one. Experiments proved that neither the window length $M$ nor the correlation time $\tau_{c}$ are determinant on their own as results are invariant for a constant ratio of these two parameters. We thus define a correlation time ratio

$$
\lambda=\frac{M}{\tau_{c}}
$$

and compute the results for several values of it.

Figure 3-a) depicts the correlation and ratio of variances profiles along the frequency axis obtained with a rectangular window. As the correlation time ratio $\lambda$ increases, the effect of correlated noise tends to vanish: the STFT real and imaginary parts tend to be decorrelated and of similar variances. However, when the length of the analysis window is of the order of the correlation time $(\lambda \approx 1)$, significant correlation between real and imaginary parts appears at midfrequencies and heteroscedaticity occurs at extreme frequencies. When the Hanning window is used (Figure 3-b)), no 

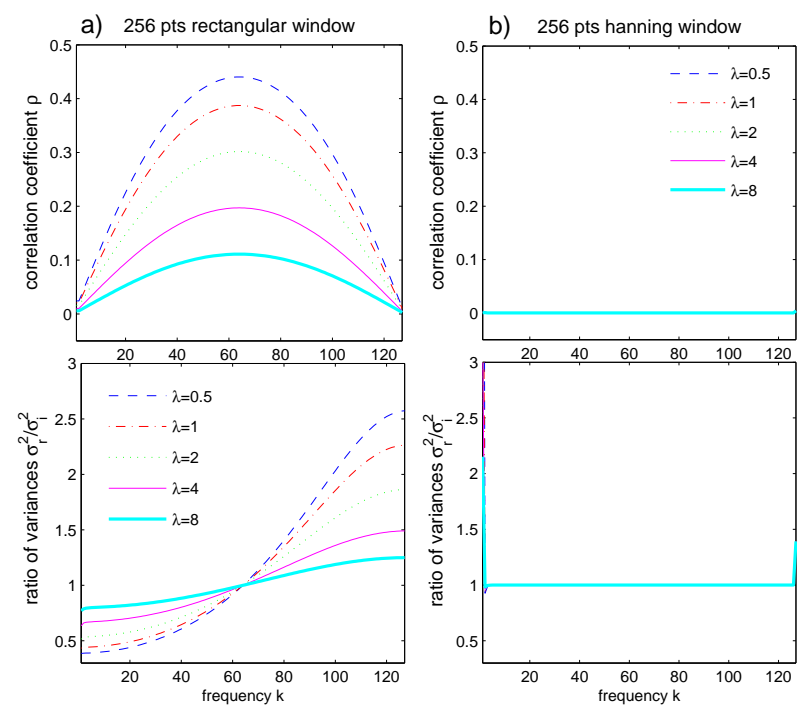

Fig. 3. Correlation coefficient $\rho$ and ratio of variances $\sigma_{r}^{2} / \sigma_{i}^{2}$ for exponentially correlated noise, using rectangular (a) or Hanning (b) window, for different correlation time ratio $\lambda$.

such phenomenon appears: the real and imaginary parts of the STFT remains uncorrelated and of similar variances regardless to the value of the correlation time ratio $\lambda$ and over all the frequency axis (except for the two first and two last frequency bins, which is a known result [1]). Results are similar for Kaiser, Blackman, Gaussian and Hamming window.

As an example, pdfs for the rectangular window at mid and extreme frequencies corresponding to $\lambda=0.5$ are observable on figure 1 (dot-dashed curves) where the dashed curve corresponds to the Hanning window. We deduce from this experiment that the choice of the analysis window impacts on the spectrogram distribution for correlated process.

\section{DISCUSSION}

The $\chi^{2}$ distribution is a well known distribution that has received extensive studies [4]. Particularly, its probability density function benefits from a known analytic formulation and methods for the estimation of its parameters. This is not the case for spectrogram distributions expressed in terms of hypergeometric functions [3]. Consequently, it may seem advantageous to approximate the spectrogram distribution with a $\chi^{2}$ distribution. We refer to the KL-divergence (section 3.2) as a measure of the quality of this approximation.

From figure 2 , a $\chi^{2}$ approximation seems perfectly reasonable if the ratio $\sigma_{r}^{2} / \sigma_{i}^{2}$ does not exceed 1.5 or when linear correlation coefficient $\rho$ lies between -0.2 and 0.2 (figures 2-b) and 2-c)). This is always the case when the Hanning window is used. Regardless to the level of correlation contained in the analysed process, the $\chi^{2}$ distribution perfectly describes the spectrogram statistics.

When a rectangular window is used, the validity of a $\chi^{2}$ approximation will depend on the ratio between the correlation time in the process and the length of the rectangular analysis window. Generally, correlation in the process under investigation is imposed. Use of long rectangular windows is thus recommended if $\chi^{2}$ distributions are used to describe spectrogram coefficients probability. This remark is in accordance with [1] where the $\chi^{2}$ law is derived under the assumption of an infinite analysis window.

In a detection task formulation, presence of signal energy at a given time-frequency location refers to the hypothesis $H_{1}$. When the noise embedding the signal is Gaussian and white, spectrogram coefficients are distributed under $H_{1}$ as a noncentral $\chi^{2}$ variable. The non-centrality parameter of the law corresponds to the spectral energy of the searched signal. The impact of a correlated noise remains to be study for this case.

\section{CONCLUSION}

In this paper, we proposed a look at the distribution of the spectrogram coefficients of a centered correlated Gaussian process. We showed that the use of a rectangular analysis window introduces correlation and heteroscedaticity between the real and imaginary parts of the STFT of the signal. We showed the impact of this phenomenon on the spectrogram distribution. We also showed that depending on the level of correlation in the analysed process and the length of the rectangular window, a $\chi^{2}$ approximation may be reasonable. For other windows like for example the Hanning one, spectrogram coefficients remains distributed as a $\chi^{2}$ law without conditions on the correlation in the process.

\section{REFERENCES}

[1] L. K. Koopman, The spectral analysis of time series, Academic Press, 1974.

[2] T.S. Durrani and J.M. Nightingale, "Probability density functions for discrete fourrier spectra," Proc. Inst. Elec. Eng, vol. Vol.120, no. 2, pp. 299-311, 1973.

[3] P.E. Johnson and D.G. Long, "The probability density of spectral estimates based on modifided periodogram averages," IEEE Trans. on Signal Processing, vol. Vol.47, no. 5, pp. 1255-1261, May 1999.

[4] N.L. Johnson, S. Kotz, and Balakrishnan, Continuous univariate distributions, vol. 2, Wiley and sons, second edition, 1995.

[5] J.P. Imhof, "Computing the distribution of quadratic forms in normal variables," Biometrika, vol. Vol. 48, no. 3, pp. 419-426, 1961.

[6] C. Hory and N. Martin, "Maximum likelihood noise estimation for spectrogram segmentation control," In Proceedings of the ICASSP02, Orlando, USA, pp. 15811584, 2002. 